\title{
Politique
}

\section{Mouvements sociaux et nouvelle culture politique}

\section{J. Yvon Thériault}

Numéro 12, automne 1987

Mouvements et acteurs

URI : https://id.erudit.org/iderudit/040566ar

DOI : https://doi.org/10.7202/040566ar

Aller au sommaire du numéro

Éditeur(s)

Société québécoise de science politique

ISSN

0711-608X (imprimé)

1918-6584 (numérique)

Découvrir la revue

Citer cet article

Thériault, J. Y. (1987). Mouvements sociaux et nouvelle culture politique.

Politique, (12), 5-36. https://doi.org/10.7202/040566ar d'utilisation que vous pouvez consulter en ligne.

https://apropos.erudit.org/fr/usagers/politique-dutilisation/ 


\title{
MOUVEMENTS SOCIAUX ET NOUVELLE CULTURE POLITIQUE
}

\author{
J. Yvon Thériault \\ Université d'Ottawa
}

Il n'existe pas aujourd'hui, dans nos sociétés, une grande synthèse philosophico-politique gouvernant l'imaginaire politique des forces contestatrices. Un discours qui, comme longtemps le marxisme le fut, donnerait à la fois l'explication des protestations (exploitation) et la direction de l'action politique (l'extension de la sphère publique). Au contraire, la plupart en conviennent, les sociétés occidentales sont aujourd'hui traversées par un éclatement des protestations sociales au fondement d'autant de discours explicatifs.

Des lectures, qui ne se résignent pas, par une prétendue loi du balancier, à voir dans la mouvance actuelle de nos sociétés un simple retour à droite, existent pourtant. La fin des avant-gardes politiques, comme l'effacement de l'image claire de la bonne société, des phénomènes provoqués par le pluralisme des acteurs sociaux, deviennent les manifestations d'une nouvelle culture politique au travail chez les forces du changement. Il apparaît dès lors possible de dégager de ce travail réalisé par les mouvements sociaux, non pas une nouvelle synthèse philosophico-politique (la nouvelle culture politique y serait allergique), mais une matrice Politique, 12 (Automne 1987). 
rendant compréhensible la trame de fond sur laquelle se déploie ce travail.

Nous croyons possible et éclairant de regrouper, sous formes de deux paradigmes, celui de «l'individualisme démocratique» et celui de "l'inscription sociale», les principales lectures voulant rendre compte aujourd'hui, comme fait social global, du pluralisme des mouvements sociaux. Deux lectures qui tantôt sont de l'ordre des présupposés théoriques, tantôt de l'ordre du constat empirique. Il ne nous a pas toujours paru nécessaire de distinguer ces deux niveaux d'analyse, voulant avant tout dresser les contours d'un questionnement au travail, peu importe pour le moment, qu'il soit actif dans le mouvement ou dans la lecture en rendant compte.

Ces deux séries de questionnement nous sont apparues contradictoires. La première repose sur les postulats normatifs de la philosophie politique et croit percevoir dans la mouvance actuelle une intensification de l'imaginaire individualiste démocratique. La seconde repose sur les prémisses sociologiques et, face à la crise culturelle des sociétés du capitalisme avancé, voit poindre la revanche des identités sociales. Deux récits dont la fusion s'avère impossible et où chacun présente, en plus d'un versant positif (émancipateur), un versant négatif. L'asocialité comme principe théorique et comme fait générateur, les nouveaux dogmatismes fondés sur les particularismes de son inscription sociale, voilà les horizons négatifs se profilant à l'avant des nouveaux mouvements sociaux. Dés apories qui ne nous semblent pas pour autant incompatibles avec la possibilité de voir, dans leur mutuelle limitation, les fondements de la nouvelle culture politique au travail actuellement dans l'apparent éclatement des forces contestatrices. C'est sur cette piste que nous conclurons cette double lecture de la signification politique des nouveaux mouvements sociaux.

\section{L'individualisme démocratique}

Le paradigme de l'individualisme démocratique est une première lecture possible de la nouvelle culture politique se dégageant 
des acteurs sociaux contemporains. Nous entendons ici par démocratie essentiellement deux choses. D'une part il s'agit d'un «état social»: la réalité d'une société se produisant à travers ses affrontements incessants (le politique). D'autre part, nous entendons par démocratie, l'idée d'une «forme politique»: le régime qui reconnaît le caractère politique de l'institutionnalisation du social et qui aménage l'espace de son déploiement. Ainsi, la démocratie n'est rien d'autre qu'un rejet conscient de toute transcendance dans l'édification de la cité (que cette transcendance soit de l'ordre d'un au-delà, d'une philosophie de la vie, d'une philosophie de l'histoire, ou plus simplement d'une tradition), pour l'exigeante acceptation du réalisme politique ${ }^{1}$. Pas de démocratie donc sans un imaginaire minimalement artificialiste sans, autrement dit, la croyance que le social est artefact. C'est pourquoi aussi la reconnaissance d'un individu libre et autonome, posé apriori comme sujet du monde (l'individualisme), est une référence essentielle à la démocratie ${ }^{2}$.

Par cette lecture les nouveaux mouvements sociaux n'ont en fait rien de nouveau. Ils ne sont que l'intensification ou le déplacement des interrogations et protestations qui naissent dans les sociétés vouées à un éternel questionnement sur elles-mêmes. Tocqueville parlait de «l'inquiète activité» à laquelle les sociétés démocratiques sont condamnées ${ }^{3}$. À la suite de ce dernier $\mathrm{C}$. Lefort insistera sur le "questionnement interminable» qui a lieu dans les sociétés où les «repères de la certitude» ont été brisés (la modernité démocratique) ${ }^{4}$. Dans les sociétés traversées par la

1. Cette définition de la démocratie est largement inspirée de la démarche de C. Lefort. Voir principalement L'invention démocratique, Paris, Fayard, 1981, et Essais sur le politique, Paris, Seuil, 1986.

2. Sur l'individualisme, comme fondement philosophique à la pensée sur la démocratie, voir PassélPrésent, «L'individu», No. 1, 1982. $1981: 340$

3. A. Tocqueville, De la démocratie en Amérique, tome 1, Paris, Flammarion,

4. C. Lefort, L'invention démocratique (op. cit.), p. 268. 
révolution démocratique aucune force ne pourrait empêcher que continuellement la domination ne soit contestée, que de nouvelles revendications pour un élargissement de l'espace public surgissent. Ce caractère effervescent de la démocratie serait plus manifeste encore dans les sociétés à forme politique démocratique (démocratie formelle) où cette dernière réalité est "génératrice» d'exigences multiples pour des droits sociaux (démocratie réelle).

L'actuel pluralisme des forces sociales et le foisonnement des interpellations démocratiques seraient donc des phénomènes virtuellement présents dans la culture politique depuis le début même de la modernité. K. Eder, en parlant des protestations actuelles, les associe d'ailleurs à une seconde vague de protestations $\mathrm{du}$ «petit bourgeois radical et démocrate» ${ }^{5}$. L'aveuglement face à la permanence de l'effervescence démocratique tirerait son origine du $19^{\text {ième }}$ siècle, qui toute imbu de «positivisme» et de croyance dans le progrès, aurait recouvert la réalité sociale d'interprétations univoques. Il est vrai que cette tendance a été favorisée, comme le souligne Habermas, par le fait que la bourgeoisie devenue à ce moment classe dominante ne sentira plus le besoin, comme à son époque ascendante, de s'appuyer sur «les valeurs universelles issues du droit naturel ou de l'éthique formaliste ${ }^{6}$. Il est vrai aussi que dans le sillage de la dérive totalitaire jacobine, la pensée progressive du $19^{\text {ième }}$ siècle accouchera, à travers le marxisme, d'une théorie du changement a-politique et anti-démocratique.

On comprend pourquoi la lecture des «nouveaux mouvements sociaux», à travers le paradigme de l'individualisme démocratique, renoue avec la philosophie politique. C. Mouffe dira à cet effet qu'il ne faut plus laisser à la droite le monopole de la réflexion philosophique sur le politique ${ }^{7}$. En effet, si le pluralisme des

5. K. Eder, "A New Social Movement", Telos, 1982: 874.

6. J. Habermas, Après Marx, Paris, Fayard, 1985: 300-301.

7. C. Mouffe, "L'offensive du néo-conservatisme contre la démocratie», in, Néo-conservatisme et restructuration de l'État, Montréal, P.U.Q., 1986: 45. 
acteurs sociaux est la caractéristique même de la modernité, c'est en interrogeant la pensée libérale-démocratique qu'on serait le mieux en position de comprendre l'éclatement actuel des forces sociales. Si ces luttes s'inscrivent dans une continuité qui les a rendues possibles, il faut alors accepter les acquis de cette continuité (la modernité) et ne pas hésiter à renouer avec une pensée prémarxiste. Car, c'est cette tradition qui, au-delà de l'historicisme et du sociologisme du $19^{\mathrm{e}}$ siècle, a reconnu le nouveau ainsi que la dimension plurielle des forces du changement. C'est toujours elle qui a perçu l'extraordinaire efficace de la référence au droit dans les sociétés où des forces contradictoires luttent pour le pouvoir mais où, aussi, aucune ne peut en toute légitimité l'occuper pleinement ${ }^{8}$.

Cette dimension démocratique (juridique) serait d'ailleurs empiriquement palpable par l'inscription actuelle des revendications féministes, ethniques, écologistes, etc., sous l'égide de demandes de nouveaux droits, tout comme, par la permanence dans l'imaginaire politique moderne de la référence aux droits de la personne. C'est toutefois par une même portée anti-autoritaire (contre le pouvoir patriarcal, contre le pouvoir étatique, contre le pouvoir scientifique, etc) que ces mouvements exprimeraient le mieux cette «crise généralisée du consentement»: autre façon de dire «effervescence démocratique».

En fait, le paradigme de l'individualisme démocratique constate plus l'effervescence démocratique qu'il ne l'explique. S'il est capable de circonscrire le contour d'une telle effervescence, il rend difficile la compréhension du sens (direction) de l'action

8. Pour ce renouveau d'intérêt en regard d'une pensée libérale du politique voir L. Ferry et A. Renaut, Philosophie politique -3-Des droits de l'homme à l'idée républicaine, Paris, P.U.F., C. Lefort, Essais sur le politique XIXe $-X X_{e}$ siècles, (op. cit.) et P. Manent, Histoire intellectuelle du libéralisme, Calmann-Levy, 1987. 
sociale contemporaine9. Faute d'une théorie critique sur la socialité contemporaine l'analyse est souvent contrainte à simplement reconnaître le nouveau tout en s'étonnant des potentialités émergentes du social (nous y reviendrons). Pour paraphraser M. Gauchet, la référence démocratique se donne difficilement «les moyens de l'exigence qui la porte»; elle se transforme difficilement en politique $^{10}$.

\section{Le fait générateur individualiste}

Cette lecture risque toutefois de nous égarer. Il est faux d'insister uniquement sur «l'inquiète activité» inhérente à la forme démocratique. Cette piste possède un versant sociologique. En effet, au delà de "l'effervescence» que la société démocratique fait naître et qui permet à la société civile d'être grosse d'une infinité de possibles, il y a une tendance, un «fait générateur», pour parler comme Tocqueville, qui traverse celle-ci. Ce «fait générateur» c'est l'individualisme inhérent autant à l'imaginaire démocratique qu'à son état social. Présent sous de multiples formes, allant de la référence universelle à l'égalité jusqu'à l'égoïste de l'homo-oeconomicus ou du narcisse moderne, il informerait toute l'activité des sociétés modernes. Autant, par ailleurs, il tend à l'atomisation du social et à l'apathie, autant il est le matériau

9. Nous ne reprenons pas ici l'analyse des limites d'une lecture démocratique en fonction de l'exigence du politique, analyse que nous avons menée dans J. Y. Thériault, "La démocratie comme politique? De la difficulté de penser le politique... à gauche"; Deuxième colloque international sur l'État, Montréal/Bishop, 1986, 37 p.

10. M. Gauchet «Les droits de l'homme ne sont pas une politique», Le Débat, juillet, août 1980: 6 . 
nécessaire aux solidarités nouvelles construites sur des arguments rationnels, sur une critique communicationnelle ${ }^{11}$.

Sous plusieurs facettes les nouveaux mouvements sociaux participeraient à cette lame de fond individualiste. E. Laclau et C. Mouffe ${ }^{12}$ n'hésitent pas à reconnaître dans la prolifération actuelle des antagonistes sociaux une intensification de «l'imaginaire égalitariste». L. Ferry et A. Renaut ${ }^{13}$, à l'intérieur de deux études récentes sur les mouvements des années soixante et des années quatre-vingt, y voient la présence nodale d'une critique antihiérarchique relevant directement de la tradition de l'individualisme radical. J. B. Elshtain ${ }^{14}$, en parlant du mouvement féministe, soulignera une forte tendance à refuser tout discours de la différence, participant ainsi à ce que $\mathrm{M}$. Gauchet ${ }^{15}$ appelle «la ligne de force de l'histoire occidentale en sa plus grande nouveauté»: «cet immense processus de dissolution de l'altérité sous toutes ses formes». Enfin, l'idée d'autonomie (comme celle plus restreinte d'autogestion), par distinction à celle de socialisme, idée centrale dans la culture politique des protestations sociales contemporaines, doit directement être associée à cet imaginaire individualiste-démocratique. L'autonomie dans son sens ethymologique «de se donner soi-même sa loi » est tributaire avant tout d'une conception de la

11. Sur l'idée du «fait générateur» chez Tocqueville voir P. Manent, Tocqueville et la nature de la démocratie, Paris, Payot, 1982. Pour une anthropologie de l'individualisme moderne, en plus des chapitres que Tocqueville lui consacre dans, $2^{\mathrm{e}}$ partie du tome II, De la démocratie en Amérique, voir les travaux de L. Dumont, Homo aequalis, Genèse et épanouissement de l'idéologie économique, Paris, Gallimard, 1977, Essais sur l'individualisme: une perspective antbropologique sur l'individu moderne, Paris, Seuil, 1983, et enfin, M. Gauchet et G. Swann, Pratique de l'esprit bumain, Paris, Gallimard, 1980.

12. E. Laclau et C. Mouffe, Hegemony and Socialist Strategy, Toward a Radical Democratic Politic, London, N.B, 1985: 160.

13. L. Ferry et A. Renaut, La pensée 68, essai sur l'anti-humanisme contemporain, Paris, Gallimard, 1985, et 68-86, Itinéraires de l'individu, Paris, Gallimard, 1987. $86 / 87$.

14. J. B. Elshtain, "Je t'apprendrai les différences», Lettre Internationale, Hiver

15. M. Gauchet et G. Swann, Pratique de l'esprit bumain, (op. cit.), p. 496. 
liberté individuelle: beaucoup moins de celle, plus sociale, de la solidarité.

Le «fait générateur» individualiste, il faut en convenir, n'est pas pour autant incompatible avec toute notion de fraternité. Suite à un récent débat avec C. Castoriadis (Pouvoirs 1986) sur l'interprétation des mouvements issus de Mai 68, Ferry et Renaut concluent, avec raison, que l'individualisme inhérent aux formes modernes de protestations n'évacuent en rien la possibilité «de nouveaux principes de lien social ${ }^{16}$. Se réclamant dans le sillage de B. Constant, d'une «liberté des modernes» ils s'opposent à l'idée selon laquelle, c'est uniquement à travers la «démocratie directe» que de tels liens puissent se nouer. Pour eux, l'horizon républicain, c'est-à-dire cette possibilité pour des sujets sociaux de transcender leurs différences et, au-delà de leurs particularismes, d'adopter un point de vue universel (solidaire) sur la cité s'avère possible dans l'univers individualiste moderne. C'est d'ailleurs, insisteront-ils, le trait dominant révélé par les revendications autonomistes actuelles: non pas celui, plus éphémère, du communautarisme utopique. Si la «démocratie directe» demeure, poursuivront-ils, une possibilité, il faut éviter (ce que les nouveaux mouvements sociaux nous enseigneraient par ailleurs) la tentation téléologique de voir dans l'imaginaire démocratique une vérité à réaliser. Les terreurs révolutionnaires étant là pour nous le rappeler, l'égalitarisme démocratique peut se muter en totalitarisme démocratique, en projet politique uniformateur. En fait, ce que Ferry et Renaut perçoivent dans les protestations contemporaines et qu'ils opposent à Castoriadis c'est la modération libérale. Cette dernière servant ici de rempart au danger dont on croyait Castoriadis prémuni, soit de transformer «l'horizon en fantasme».

16. L. Ferry et A. Renaut, 68-86 Itinéraires de l'individu, (op. cit.) p. 43. Les contributions au débat sur la portée individualiste des mouvements issus de mai 68 sont présentées dans Pouvoirs, No 39, 1986. La réponse de Ferry et Renaut à la critique de Castoriadis est insérée dans le livre, 68-86... p. 49 et ss. 
Mais, et c'est ce qui est important pour le débat qui nous préoccupe, au delà de ce refus de la forme représentative moderne au nom d'une démocratie totale, directe, à l'ancienne (le rejet de la modernité), il s'agit bien dans les deux cas d'une même conception constructiviste du lien social. La nouvelle querelle des anciens et des modernes repose, en définitive, sur le postulat individualiste démocratique. C'est la présence ou non de l'argumentation, de la construction d'une pratique collective à travers la communication critique (non la solidarité comme fait social — nous y reviendrons) qui signe la dimension fraternelle des mouvements contemporaines. Que cette dimension soit appréhendée à travers l'idéal républicain (Ferry et Renaut) ou à travers celui de la démocratie directe (Castoriadis) ${ }^{17}$, la solidarité comme artefact demeure l'horizon indépassable du paradigme.

À l'intérieur d'une lecture plus sociologique, cette culture de l'argumentation, au centre de la nouvelle texture politique, a été aussi notée. Des analyses nombreuses, en partant du constat du caractère «transfonctionnel» et «transclassiste» ${ }^{18}$ des mouvements, ont sont venues à rejeter les prémisses de la sociologie classique: soit de chercher les "conditions structurelles» de la genèse de l'action collective. L'idée du mouvement personnage, pour reprendre l'expression de Melucci, et ses postulats de causalité structurelle, ne peut rendre compte de la diversité interne retrouvée à l'intérieur de ces mouvements. Au contraire, il faudrait inverser l'ordre du raisonnement, refuser toute approche «essentialiste»

17. L'idée de la démocratie chez Castoriadis est présentée dans, «La polis grecque et la création de la démocratie, Le Débat, janvier-mars 1986. L'idée d'autonomie et de démocratie directe dans l'interprétation des mouvements contemporains, particulièrement mai 68, est brossée par C. Castoriadis dans «Les mouvements des années soixante», Pouvoirs, No. 39, 1986: 107-116 et dans Mai 68: La brêche (avec E. Morin et C. Lefort), Paris, Payard, 1968.

18. Les termes «transfonctionnels» et «transclassistes» sont utilisés par F. Feller et A. Heller pour caractériser le type de regroupement des nouveaux mouvements sociaux. Voir, "From Red to Green", Telos, 59, 1984: 35. 
(Laclau et Mouffe), chercher plutôt comment des «espaces de rassemblement d'acteurs» (Melucci) éclairent des situations structurelles de subordination et les transforment en antagonismes sociaux ${ }^{19}$. Une lecture de la structuration de l'action collective, quoique plus conflictuelle, qui n'est pas sans rappeler les approches américaines de «mobilisation de ressources» élaborées aussi, autour des années soixante, pour rendre compte de la présence d'une action rationnelle chez les acteurs sociaux. Et ceci, face à une compréhension, à l'époque prédominante, essentiellement behaviouriste des rassemblements collectifs ${ }^{20}$.

Ainsi, il ressort de telles analyses qu'une communication critique, une culture égalitaire démocratique, est à la source des regroupements sociaux revendicateurs. Autrement dit, avant d'être femme, jeune, minoritaire, soumis au danger de la guerre nucléaire ou à la pollution industrielle, l'acteur social est traversé par la culture de l'argumentation critique. C'est essentiellement une telle lecture qui signerait l'horizon solidaire de ces mouvements: la fondation de sociabilités discursives. Et, pour qu'une telle rationalité stratégique existe il faut, autant au niveau de la pensée qui en rend compte que de la pratique, qu'un individu faisant

19. Les références à Melucci sont puisées de «Mouvements sociaux, mouvements post-politiques", Revue international d'action communautaires, 10/50, 1983: 13-30 et "The Symbolic Challenge of Contemporary Movements", Social Research, 52, 4, 1985: 789816. Ces deux articles sont nettement plus axés vers la dimension «rassemblement d'acteurs», par opposition à mouvement exprimant les contraintes structurelles des systèmes, que ses textes précédants: voir par exemple, "Société en changement et nouveaux mouvements sociaux", Sociologie et Société, 10, 2, 1978: 37-54. E. Laclau et C. Mouffe, Hegemony and Socialist Strategy... (op. cit.) proposent une critique voisine de ces approches théoriques fondées sur une lecture «essentialiste» des mouvements sociaux.

20. J. Cohen, «Strategy or Identity: New Theoretical Paradigms and Contemporary Social Movements", Social Research, 52, 4, 1985: 663-716, présente une intéressante synthèse de ce qu'elle appelle l'approche de la «rationalité instrumentale stratégique» dans laquelle elle insère les théoriciens de la "mobilisation des ressources». On trouve aussi chez Pizzorno dans, "On the Rationality of Democratic Choice» Telos, 63, 1985 : 41-69, une critique des approches trop fortement marquées, à son avis, par la rationalité individualiste démocratique, approches qu'il associe à des conceptions néo-utilitaristes. 
usage de sa raison soit postulé et se croit au fondement de la construction du social.

Les apories de l'individualisme démocratique: l'asocialité

L'individualisme, autant pratique que théorique, inhérent au paradigme comporte toutefois des effets jugés corrosifs pour l'action collective. Dans un premier temps le radicalisme démocratique, par sa conception construtiviste du monde - assemblons nous et formons une société - , peut facilement conduire à l'oubli des contingences du social, à la négation d'une inscription dans l'histoire. Nous l'avons souligné, par d'imaginaire démocratique sans la référence à un point zéro de sociabilité, sans la fiction de l'égalité première, de la non différentiation originelle. Ce sont les postulats de la communication critique, ceux rendant possible le renversement de toutes relations de subordination ou de différence en rapport antagoniste. Mais ce point d'archimède, nécessaire, sert aussi au nom d'une vérité première (l'égalité) à nier toute historicité.

J. B. Elshtain ${ }^{21}$, en réfléchissant sur certains courants dominants du féminisme américain, démontre bien cette tendance. Pour elle, l'histoire des femmes, racontée à partir du postulat de la non-différenciation originelle, a souvent masqué l'histoire concrète des relations hommes/femmes. On est ainsi conduit à ne percevoir dans cette histoire qu'une séculaire subordination des hommes sur les femmes au détriment de toutes les autres formes d'inscriptions que cette relation a historiquement fait naître. Cette lecture s'avérant, par le fait, incapable de penser les différences (pourtant fortement présentes dans la conscience des femmes). Ce point de vue arrive aussi, en s'appuyant sur l'idée toute démocratique de

21. De J. B. Elshtain voir «Je t'apprendrai les différences», (op. cit.) et Public Man, Private Woman, Princeton, Princeton University Press, 1981. 
l'illégitimité de toute différence, à concevoir «l'identité sexuelle comme une prison», à rejeter toute réalité fondée sur une différenciation des genres.

Ce qu'elle appelle la «revendication androgyne» s'avère tout aussi incapable d'imaginer l'avenir autrement que par une projection de ce vide premier, ou encore, par une invitation aux femmes à rejoindre l'univers rationnel mâle. C'est l'idée d'un état de nature identitaire, d'une table rase matrice du monde, rappelle Elshtain, qui explique «l'idéologie anti-famille» massivement présente dans le féminisme et qu'elle n'hésite pas à associer au fantasme totalitaire d'un monde identitaire, sans distinction aucune entre le public et le privé ${ }^{22}$. De toute façon, l'avenir ne pourrait être féminin: l'idée d'un monde «où les sexes peuvent jouer des rôles infiniment interchangeables» n'étant pas fondée sur l'histoire de la différence mais bien sur «la perspective téléologique de la neutralité sexuelle» ${ }^{23}$. Le deuxième sexe de $\mathrm{S}$. de Beauvoir, comme l'ouvrage de Dialectic of Sex de F. Firestone sont des œuvres, ajoute-t-elle, marquantes de cette lecture. Des récits qui condamnent la femme à adopter le discours universel, rationnel (mâle), le discours de la civilisation ou à demeurer extérieur à la Civilisation ${ }^{24}$. Comme le dit autrement A. Leclerc ${ }^{25}$ en commentant le livre de Belotti $D u$ côté des petites filles: «tout ce qui passe pour masculin se trouve immédiatement valorisé, tout ce qui passe pour féminin immédiatement déconsidéré, rejeté, dédaigné; comme si ça allait de soi».

Cette perspective a-social n'est pas uniquement présente dans les lectures réalisées sur ou par les acteurs démocratiques. Les apories du paradigme se retrouvent aussi dans la «sensibilité moderne», par la présence de cette culture «narcissique» que $\mathrm{C}$.

22. J. B. Elshtain, Public Man, Private Women, (op. cit.), p. 335.

23. J. B. Elshtain, "Je t'apprendrai les différences», (op. cit.).

24. J. B. Elshtain, Public Man, Private Women, (op. cit.), p. 308.

25. A. Leclerc, Homme et femme, Paris, Grasset, 1985: 196. 
$\mathrm{Lash}^{26}$ décrit, pour l'Amérique de la fin des années soixante-dix, comme une dérive pathologique de l'action sociale de la décennie précédente. Un individualisme hédoniste, un culte effréné du moi, une culture politique où la dimension collective est incapable de se frayer une voie.

On connaît depuis Tocqueville, et même plus tôt dans les écrits par exemple du jeune Marx, cette tendance à l'atomisation caractéristique de la modernité. Sous cet aspect, l'individualisme économique du libéralisme anglais (de Locke à Bentham) ne serait que la reconnaissance d'un phénomène sociologique plus vaste, soit celui de l'émergence sous de multiples facettes de l'individu moderne. Les sociétés démocratiques ne font pas que reconnaître l'individu comme étalon: elles sécrètent l'individualisme. L'image de l'individu dans «la foule solitaire» est trop présente pour que nous ayons à développer longuement cette continuelle brisure du lien social qu'opère l'état social démocratique. Rappelons simplement cette phrase de Tocqueville: «L'individualisme est un sentiment réfléchi et paisible qui dispose chaque citoyen à s'isoler de la masse de ses semblables et à se retirer à l'écart avec sa famille et ses amis; de telle sorte que après s'être ainsi crée une petite société à son image, il abandonne volontiers la grande société à elle-même» ${ }^{27}$. Au départ simplement délétère sur les "vertus publiques", l'individualisme est toujours susceptible de s'attaquer à toutes les valeurs liant les hommes entre eux, bref à s'absorber dans «l'égoïsme».

La complexité de l'indiviualisme d'ailleurs est présentée dans toute son ambiguïté par G. Lipovetsky dans L'ère du vide. Si, plus que tout autre, il perçoit dans la mouvance sociale actuelle une phase nouvelle, une intensification de la «révolution démocratique conçue comme dissolution de l'univers hiérarchique et avénement

26. C. Lash, Le complexe de Narcisse, Paris, Lafont, 1979.

27. A. Tocqueville, De la démocratie en Amérique, (op. cit.), p. 125. 
du règne de l'égalité» ${ }^{28}$; il y voit aussi «la personnification narcissique, la fragmentation disparate du moi, l'émergence d'un individu obéissant à des logiques multiples à l'instar des juxtapositions compartimentés des artistes pop ou des combinaisons plates et aléatoires d'Adami» ${ }^{29}$. On ne sait pas, à la lecture de Lipovetsky, si ce "vide», cette déambulation existentielle de l'individu moderne est un effet salutaire ou pervers, une tendance ou une nécessité de la révolution démocratique. Toutefois, il est clair qu'un tel regard permet de voir l'effervescence des acteurs sociaux sous un œil inquiétant. Loin d'ouvrir de nouveaux champs d'interpellations démocratiques la prolifération des mouvements sociaux ne serait que la démonstration des effets négatifs (dissolvants) de l'individualité démocratique sur l'action collective. Un pluralisme social qui exprimerait la paralysie de l'action dans les sociétés du relativisme absolue, où chaque interpellation est susceptible d'une égale vérité. Des effets de mode: une multiplicité chimérique d'acteurs et de mouvements sociaux qui braquent momentanément leur regard furtif sur un enjeu, ne faisant ainsi que "parachever la logique de l'indifférence».

\section{L'inscription sociale}

Le second paradigme par lequel peut être appréhendé la signification politique des nouveaux mouvements sociaux est d'inspiration plus proprement sociologique. Les acteurs sociaux sont inscrits à l'intérieur d'une réalité sociale qui les dépasse et qui structure leur action. Ce n'est pas l'individu, ni même l'acteur qui est premier; la société à travers ses règles, ses réseaux de relations sociales et ses rapports de pouvoirs, acquiert un statut

28. G. Lipovetsky, L'ère du vide, essais sur l'individualisme contemporain. Paris, Gallimard, 1983: 219.

29. Ibid., p. 125. 
ontologique. Le sens des luttes sociales est tiré de l'inscription singulière de l'acteur dans une histoire. Il s'agit donc, avant tout, de déchiffrer la vérité de cette inscription pour connaître l'orientation normative présente ou à donner à l'action sociale.

Une première version, macro-sociologique, de cette lecture existe à travers la thèse de la société... post-industrielle, capitaliste avancée, ou de la société programmée. Les nouveaux mouvements sociaux expriment, dans ces sociétés, les enjeux issus de la nouvelle structuration du pouvoir. Que l'on parle de l'avénement d'un nouveau système d'action historique (société industrielle/société programmée - Touraine) ou d'une étape dans l'évolution de la modernité capitaliste (le capitalisme avancé - Habermas), toujours il s'agit d'annoncer une rupture sociologique explicative de l'existence de la configuration actuelle des acteurs sociaux. C'est ici d'ailleurs que le vocable «nouveaux mouvements sociaux» tire son origine et prend son sens. L'acteur historique de la société industrielle ou du capitalisme concurrentiel - le mouvement ouvrier prototype du mouvement social - se voit supplanté par l'émergence d'une pléiade de regroupements collectifs exprimant la nouvelle donne de nos sociétés.

Chez Habermas ${ }^{30}$, pour contrer les crises structurelles du capitalisme concurrentiel $\left(19^{\mathrm{e}}\right.$ siècle), ainsi que pour satisfaire les exigences d'une plus grande égalité et justice distributive issues de la pensée universelle et formaliste bourgeoise, on a progressivement assisté à une prise en charge des institutions sociales et de la société civile par l'État (État-social). L'État devant dorénavant assurer les tâches contradictoires de la régulation économique et du maintien, à travers les prestations sociales, de la loyauté des masses. Les crises, de directement économique qu'elles étaient

30. C'est dans Raison et Légitimité, Paris, Payot, 1978 que Habermas présente une analyse systématique des crises des sociétés du capitalisme avancé et des problèmes de légitimation qui en découlent. 
(exprimées par le conflit de classe), seront progressivement déplacées à l'intérieur du système politico-administratif (crise de la rationalité - crise de légitimation) et, plus tardivement, dans le système socio-culturel (crise de motivation). Cette nouvelle réalité du capitalisme avancé correspond à une intensification de l'effritement du monde vécu socio-culturel (lieu d'expression de la normativité sociale), espace continuellement colonisé par les logiques rationnellepar-rapport-à-une-fin des systèmes économiques et politiques. Les crises de légitimité et de motivation s'expriment par la défense problématique de son identité sociale, par la recherche d'une «bonne-vie», par l'interrogation sur «la grammaire des formes de vie» ${ }^{31}$. La question des identités, si elle est nécessairement plurielle, apparaît souvent pour Habermas une simple réaction défensive face à l'instrumentalisation de la vie et, par conséquent, n'est pas nécessairement progressive - nous y reviendrons.

La dimension plus proprement culturelle de l'action sociale contemporaine est aussi fortement rappelée par A. Touraine. Pour celui-ci, la société dans laquelle nous entrons, la société programmée, organise le pouvoir autour de la capacité des grands appareils à gérer et à monopoliser l'information. C'est une société où le contrôle de l'organisation du travail est moins central que le contrôle des connaissances par lesquelles sont programmés les appareils de production et d'information. Une société condamnable avant tout parce qu'elle opprime à travers la manipulation des consciences et non plus principalement, comme la société industrielle, parce qu'elle exploite à travers le procès de travail. Les nouveaux mouvements sociaux sont les acteurs centraux de la société programmée en raison de leur opposition, à partir d'une

31. Voir Habermas, «New Social Movements», Telos, 48, 1981: 33-37. 
identité sociale et au nom de l'innovation culturelle, au modèle culturel de gestion des technocrates ${ }^{32}$.

Il faut ici faire une parenthèse. On ne peut insérer Touraine du côté d'une analyse plus structurelle, substantive, de la réalité sociale, sans justifier un tel choix. N'est-il pas celui qui ne cesse de clamer la primauté de l'action sur la structure, celui qui applaudi la reconnaissance tardive du sujet dans le retour, au centre de l'analyse, de l'acteur. Rappelons simplement comment l'action sociale chez lui est inscrite dans un champ qui culturellement l'oriente. Son travail, à notre avis, ne se comprend que lu à travers une priorité ontologique donnée aux contours de l'historicité, donc aux structures des sociétés programmées ${ }^{33}$. Ce n'est qu'à l'intérieur d'une telle tentative qu'il lui est possible de préciser les limites à une lecture «démocratique» et «libérale» de l'action collective. En effet, les sociétés programmées ne sont pas vouées à l'indécision démocratique ${ }^{34}$. L'action des mouvements sociaux a un sens qui lui est donné par l'historicité dans laquelle ils s'inscrivent. Lorsque l'action conflictuelle se rapproche le plus des enjeux centraux du système d'action historique (historicité), le

32. Des nombreux travaux de Touraine, voir principalement sur les mouvements sociaux et la société programmée, La voix et le regard, Paris, Seuil, 1978. L'après socialisme, Paris, Grasset, 1980, et Le retour de l'acteur, Paris, Fayard, 1984.

33. C'est une interprétation similaire, il nous semble, que propose L. Maheu dans "Rapports de classes et problèmes de transformation: la thèse de la société postindustrielle», Sociologie et sociétés, 10, 2, 1978: 11-36 et dans, "Conflits et mouvements sociaux: enjeux et caractéristiques de la domination culturelle et politique», 1986: 53 p., Deuxième colloque international sur l'État, Montréal/Bishop. Touraine s'inscrirait dans une continuité avec les analystes des classes sociales et ceux voulant définir les enjeux structurels des sociétés industrielles. Ainsi, J. Cohen, dans «Between Crisis Management and Social Movements: The Place of Institutional Reform»Telos, 52, 1982: 33 et ss, reproche, à tort il nous semble, à Touraine d'être incapable de saisir la dimension structurelle de l'insertion des mouvements sociaux, parce qu'influencé problématique par la sociologie de l'acteur. L'analyste systémique dans laquelle Touraine insère son modèle culturel n'apparaît pas pour Cohen le centre de sa méthode.

34. Voir sur cette question la dernière section de Le retour de l'acteur (op. cit.) où sont discutés les rapports entre démocratie (représentativité) et mouvements sociaux. 
mouvement social est à son niveau le plus élevé de participation à la production de la société par elle-même. Toute la démarche tourainienne de «l'intervention sociologique», méthode proposée pour appréhender le sens le plus noble (élevé) de l'action des mouvements sociaux, ne peut que reposer sur l'existence d'une telle vérité normative, extérieure au mouvement.

Cet «étalon» Touraine le puise par ce qui peut paraître, selon ses propres prémisses, une double régression. D'une part, il réinsère son analyse dans une lecture «évolutionniste» (société agraire, société marchande, société industrielle, société programmée) où chaque étape implique une plus grande capacité (parce que plus consciente) de la société à produire sa propre historicité ${ }^{35}$. Et, deuxièmement, comme la sociologie classique, il cherche dans la matrice culturelle de l'institution du social le code explicatif de l'action collective (le travail dans la société industrielle, l'innovation culturelle dans la société programmée). C'est au prix de ces régressions problématiques qu'il se donne toutefois les moyens de chercher, au delà de la fragmentation, le principe articulatoire à l'exigence de l'action politique.

\section{Les sociabịlités diffuses}

Car, ce que révèlent principalement le paradigme de l'inscription sociale et la thèse de la société post... industrielle, capitaliste, moderne, est, face à la rationalisation du monde, la revendication plurielle d'identité ${ }^{36}$. Contre la marchandisation, l'instrumentalisation, la technocratisation, la bureaucratisation,

35. Sur le néo-évolutionnisme de Touraine, voir L'après socialisme (op. cit.) p. 107 et ss. K. Eder, dans "A New Social Movements» (op .cit.) et J. Cohen, dans «Between Crisis Management and Social Movements» (op. cit.) insistent aussi sur le caractère "néoévolutionniste» de l'analyse tourainienne et ceci malgré les constantes critiques adressées par Touraine à l'évolutionnisme.

36. C'est à l'espace théorique réservé à ces sociabilités dans la tradition marxiste que s'intéressait l'analyse que nous avons menée dans La société civile, ou la chimère insaisissable, Montréal, Québec/Amérique, 1985. 
et au nom d'une spécificité culturelle, des acteurs sociaux, mobilisant ce qui leur reste de forme de vie, s'activent. Touraine dira à cet effet: "la contre partie du refus de l'État, de la crise culturelle et de la conscience angoissante du vide social est la recherche de l'identité personnelle et de la communauté capables de résister aux déchirements de l'histoire " 37.

C'est pourquoi, par ailleurs, la pensée d'Illich est, pour lui, «la grande utopie de notre temps». Celui, poursuivra-t-il, que cette pensée "laisse indifférent ou hostile ne trouvera pas la route qui conduit vers les nouveaux mouvements sociaux de la société programmée » ${ }^{38}$. Par conséquent, il lui apparaît encore plus urgent de se prémunir contre le double écueil auquel nous conduit cette utopie: le danger qu'elle se substitue à la tâche première des mouvements sociaux actuels, la lutte anti-technocratique; et le danger du retrait communautaire avec toute sa charge anti-politique. L'exigence d'un principe unificateur visant à pallier à la fragmentation et au pluralisme que suscitent les appartenances communautaires diffuses devient ici évident.

L'idée de la riposte des sociabilités diffuses face à la prégnance du système est aussi au centre de l'analyse, proposée par C. Offe ${ }^{39}$, de l'innovation politique annoncée par les nouveaux mouvements sociaux. S'inscrivant à l'intérieur d'une démarche apparentée à celle de Habermas, Offe s'intéresse à la crise de légitimité née des exigences contradictoires dirigées vers le système politique. Les nouveaux acteurs sociaux, soulignera-t-il, agissent au nom de «collectivités ascriptives»: âge, sexe, race, région, ou encore, comme c'est le cas pour les pacifistes et les environnementalistes, au nom de la race humaine dans sa totalité. Ils problématisent

37. A. Touraine, La voix et le regard (op. cit.), p. 31.

38. Ibid, p. 32 .

39. C. Offe, «New Social Movements: Challenging the Boundaries of Institutional Politics», Social Research, 52, 4, 1985: 817-868. 
des champs nouveaux laissés en friche par l'ancienne gauche: le territoire, l'espace, le monde-vécu, le corps, la santé, l'identité sexuelle ${ }^{40}$. Ce sont donc des mouvements substantifs qui opposent à la logique des systèmes des orientations normatives puisées dans des particularismes sociaux. Ce contenu normatif, rappelle ailleurs $\mathrm{K}$. Eder, est souvent emprunté au fond romantique et populiste qui sert, depuis fort longtemps, à nourrir la critique antirationnelle du capitalisme ${ }^{41}$.

\section{Les apories de l'identité sociale: les passions communautaires}

C'est ici d'ailleurs que le paradigme de l'inscription sociale pose, sur la dimension politique de ces mouvements, une série de questions. Du moins cette lecture se présente sous forme contradictoire avec celle brossée préalablement à partir du paradigme de l'individualisme démocratique. Ici, la solidarité n'est plus postulée construite, artefact; elle est un fait premier qui englobe les consciences individuelles et s'impose comme vérité. Pour reprendre une distinction proposée par Feher et Heller, on ne retrouverait pas nécessairement dans la mouvance actuelle une culture de la «communication critique» mais bien souvent une culture "symbolique iconographique» ${ }^{42}$. Ces auteurs croient d'ailleurs noter, dans l'évolution récente des mouvements sociaux, une tendance dans ce sens ( «From Red to Green»): un passage des thèmes de la liberté (anti-autoritarisme-droits civils) à des thèmes de la vie (écologie, paix, avortement). Les slogans popularisés en Allemagne, «mieux rouge que mort», "rien n'est aussi important

40. Ibid, p. 839.

41. K. Eder, «A New Social Movement» (op. cit.), "The 'New Social Movements': Moral Crusades, Political Pressure Group, or Social Movements?», Social Research, 52, 4, 1985: 869-889.

42. F. Feller et A. Heller «From to red to green» (op. cit.). 
que la vie», expriment assez bien la dévalorisation ainsi réalisée du politique. Des observations qui rejoignent nombre d'analystes notant, dans les revendications fondées sur l'identité, un fort contenu normatif à caractère parfois régressif ${ }^{43}$. Bref, des acteurs légitimant leur action en référence à une culture «fondamentaliste» ou "communautariste» fort éloignée sinon antinomique de la culture rationaliste démocratique. Car, pour utiliser une formule de Baechler: «la communauté est, par sa nature même, le lieu des passions et non celui de la raison ${ }^{44}$.

Paradoxalement, si au centre de la lecture de l'inscription sociale se trouve l'idée de la rupture avec la société capitaliste industrielle (nouvelle structuration et nouveaux acteurs sociaux), la perspective politique qui s'en dégage repose sur les postulats classiques du sociologisme (ou même du transcendantalisme): l'action politique vise à réaliser une vérité inscrite dans le social (ou méta-sociale). Pour le dire autrement, alors que les penseurs de l'individualisme démocratique croient découvrir à l'intérieur des mouvements contemporains un retour à une éthique de la responsabilité, le portrait tiré d'une lecture de la crise des sociétés du capitalisme avancé nous fait voir de «nouvelles» éthiques de la conviction. Les acteurs n'interviennent pas pour arriver par la discussion à des consensus provisoires: contre le vide d'un monde réifié, ils parlent au nom d'une vérité révélée par leur singulière inscription dans une histoire.

R. Senett a appelé Gemeinschaft destructrice la culture intimiste propagée par la «nouvelle gauche» américaine autour des années soixante. Une volonté de projeter, comme modèle d'espace public,

43. A. Melucci dans ses premiers travaux, "Société en changement...» (op. cit.), notait ce phénomène. $\mathrm{K}$. Eder, dans les textes précédemment cités ainsi que J. Cohen "Strategy or Identity...» (op. cit.) et dans «Rethinking social movements» Berkely Journal of Sociology, 29, 1983, se réferent souvent au contenu "fondamentaliste», "quasi religieux», de la culture de plusieurs néo-mouvements sociaux.

44. J. Baechler, Démocraties, Paris, Calman-Lévy, p. 161. 
les rapports chauds, étroits, des communautés. Une perspective politique qui vise à effacer la distinction privé/public, en faisant du public une simple extension du privé. Un processus qui à terme conduit à la perte de tout espace public: c'est-à-dire de lieu où l'individu, distancié de son accrochage communautaire, peut jouer le rôle de citoyen dans un espace où par des règles on a établie «une égalité initiale fictive entre les joueurs» ${ }^{45}$. Car, rappelle toujours Senett, une vie publique active, fondée sur le changement, ne peut être pensée sur le modèle d'une communauté affective. Dans ce sens "l'homme qui change 'trahit' la communauté ${ }^{46}$. Celui qui croit faire l'histoire par la discussion rejette son héritage social. Seule la vision d'une "démocratie directe» fondée sur une communion parfaite entre l'individu et sa communauté demeure ainsi compatible avec une telle représentation intimiste de l'espace public. Mais... parle-t-on alors toujours de démocratie.

Les remarques précédentes peuvent d'ailleurs être appliquées aux appels à la convivialité d'Illich. Par exemple, la reconnaissance chez-lui des genres, si elle est salutaire par rapport à une perspective abstraite de la neutralité sexuelle, ne saurait reconnaître l'extraordinaire travail d'égalisation et de communication critique réalisé par la révolution démocratique et son processus d'individualisation ${ }^{47}$. Il parle ainsi exclusivement à des femmes et non à des citoyennes. Il ne lui reste alors qu'à proposer à celles-ci, pour éviter l'univers sexiste non genré, un retour à leur communauté de femmes: celle pré-moderne insérée dans une hiérarchie des genres. En fait, au delà de l'appel illichéen, du lesbianisme politique au «real women»,

45. R. Senett, Les tyrannies de l'intimité, Paris, Seuil, 1979, p. 214.

46. Ibid, p. 246.

47. I. Illich, Le genre vernaculaire, Paris, Seuil, 1982. On lira avec intérêt la virulente attaque que dresse Marie-Jo Dhavernas contre ce livre dans «Illich ou la clémence d'Auguste", La revue d'enfance, Automne 84, No. 14. Cette critique, il va sans dire, est essentiellement fondée sur une perspective de neutralité sexuelle originelle. 
en passant par le postulat féministe plus largement répandu d'un langage théorique autre parce que porté par son vécue de femmes, un large pan du féminisme apparaît traversé par cette référence au "genre», à la spécificité, comme porteur d'un point de vue vrai, «iconographique», "non discutable» sur le social ${ }^{48}$.

Dans le portrait du nouveau paradigme politique qu'il dresse C. Offe est très explicite sur les tendances anti-discursives présentes dans les modes d'action des nouveaux mouvements sociaux. Leur tactique, précise-t-il, ne passe plus prioritairement par les canaux représentatifs politiques traditionnels. Elle vise plutôt à mobiliser l'attention publique à travers des moyens (grossièrement) légaux et non-conventionnels. Leur discours est truffé de formes grammaticales qui laissent peu de place à la complexité, à la nuance, à la négociation, au compromis: concepts inhérents à l'idée même du politique. «Jamais», «nulle part», «cessez», «gelez», «banissez», ou des antinomies telles «oui/non», «eux/nous» «maintenant ou jamais», sont les slogans, dit-il, prisés par la nébuleuse politique actuelle. Ils mettent l'emphase sur le non négociable et les questions de principes $^{49}$.

Et, en effet, on ne peut négocier des questions considérées aussi vitales: le pacifisme insiste sur "l'apocalypse now» ou le «no futur"; l'écologisme sait que les maux de notre société sont redevables à l'attitude prédatrice vis-à-vis la nature au fondement de notre société technicienne; le féminisme parle d'une position universelle de victime qui confirme la pureté du discours et qui rend illégitime toute argumentation critique; le militant nationalitaire révèle une vérité, celle de la nation ou de l'ethnie, au dessus des aléas du politique. Toute hésitation sur des prémisses aussi nobles est dès lors considérée comme une faiblesse, un pacte avec l'ennemi.

48. Voir les critiques dans ce sens formulées par J. B. Elshtain, "Je t'apprendrai les différences", (op. cit.) et Public Man. Private Woman. (op. cit.).

49. C. Offe «New Social Movements..." (op. cit.). p. 830 et ss. 
La difficulté qui naît de ces inscriptions singulières, c'est que chacune a sa manière, à partir de sa position "privilégiée» prétend tenir le discours vrai sur le social, le code permettant enfin d'évacuer le politique. On ne rompt pas avec l'idée du sujet historique porteur d'un message et d'une pratique universelle de libération. C'est la projection de sa spécificité (de victime, de minoritaire), celle du lieu d'où l'on parle (au nom de la nature, au nom de la vie humaine) qui, ici, est perçue comme allant réaliser cet universel: non pas, comme dans l'individualisme démocratique, l'appel a une identité originelle. Des sectarismes partiels auraient remplacé le sectarisme d'une classe. Et, encore ici, dans cette mutation plus personne ne prétend parler au nom des intérêts collectifs immédiats: plus de sujet se définissant, simplement, comme citoyen participant à la chose publique. Des pratiques «actuelles» séparatistes devant réaliser "plus tard» l'humanité nouvelle.

Les analystes des mouvements sociaux, ceux s'intéressant à la nouvelle configuration politique révélée par ce foisonnement des identités (non pas ceux acceptant la lecture singulière d'un mouvement précis), sont d'ailleurs très réticents à avaliser de telles tendances particularistes. C'est ainsi qu'ils sont, soit contraints à poser un jugement négatif sur la portée progressive des nouveaux acteurs sociaux, soit obligés de rompre avec une perspective essentiellement sociologique. Dans ce dernier cas il s'agit de réinsérer dans l'analyse une dimension subjective (l'identité fondée sur la communication critique) dont la présence empirique ou non signe le caractère émancipateur du mouvement.

A. Touraine, on l'a souligné, dénonce le danger du repli communautaire et cherche le principe unificateur qui rallierait les diverses identités contestatrices de la société programmée. En se référant, pour jauger de la portée innovatrice d'un mouvement social, à son inscription dans un cheminement où l'acteur atteint 
un niveau plus élevé d'une conscience de production de la société, il fait appel, à un critère qu'il puise dans l'imaginaire rationaliste de la modernité, non à un sens extirpé de la pratique sociale ellemême. Les analyses spécifiques réalisées dans le cadre de «l'intervention sociologique» ont d'ailleurs été généralement négatives sur le constat de la présence effective de telles visées dans les pratiques concrètes des mouvements.

Chez Habermas aussi on trouve une distance entre le projet possiblement émancipateur des mouvements sociaux et le constat empirique des pratiques conflictuelles. Dans ses premières évaluations des nouveaux mouvements sociaux (principalement le mouvement étudiant), il y percevait une amorce de démocratisation contre le projet technocratique visant à fonctionnaliser les institutions sociales et l'espace public. Plus tardivement, il y verra plutôt des réactions systémiques, essentiellement défensives, face à l'instrumentalisation du monde vécu socio-culturel. Ces mouvements étant alors caractérisés par leur orientation particulariste et leur action normative. Ce n'est que très récemment, à travers un approfondissement de sa théorie de la communication dans la logique de l'évolution des processus cognitifs, qu'il croit percevoir dans certaines pratiques actuelles (principalement à l'intérieur du féminisme) les prémisses d'une action communicationnelle ${ }^{50}$. Autrement dit, là existeraient les virtualités d'une pratique s'inscrivant dans la reconnaissance des fondements universels des normes et des valeurs.

50. Voir, Thérie de l'agir communicationnel, 2 tomes, Paris, Fayard, 1987. Certains éléments de la théorie communicationnelle d'Habermas sont présentés dans Après Marx (op. cit.). On y trouvera l'évaluation virtuellement positive du féminisme en regard d'une communication critique (p. 59). Enfin J. Cohen dans "Strategy or Identity" (op. cit.) et J. Cohen et A. Arato dans "Civil Society and Social Theory", Deuxième colloque international sur l'État, Montréal/Bishop, 1986, 44 p., retracent le cheminement de la pensée d'Habermas en regard des nouveaux mouvements sociaux. 
Enfin, C. Offe abonde dans un sens tout à fait similaire. Ses premières analyses des crises du système politique du capitalisme avancé le conduisaient à nier tout potentiel non systémique (innovateur) aux nouvelles forces contestatrices. Aujourd'hui, en distinguant à l'intérieur des mouvements sociaux deux courants principaux de critique de la modernisation technocratique, il y perçoit un potentiel progressif. Les classes moyennes (les porteuses pour Offe des nouveaux mouvements sociaux) sont scindées entre l'ancienne classe moyenne qui fonde sa critique sur une «normativité irrationnelle», «pré-moderne», «particulariste» et, la nouvelle classe moyenne qui fonde son argumentation sur des valeurs «émancipatrices», «universalisables", sur la "capacité cognitive avancée» de la modernité ${ }^{51}$ Chez lui c'est aussi, on comprendra pourquoi, essentiellement autour de cette nouvelle classe moyenne (et du mouvement ouvrier) que la reconstruction d'un paradigme politique de gauche s'avère possible.

Bref, pour contrer les tendances «particularistes» révélées par la lecture de la crise des sociétés du capitalisme avancé, les analystes doivent emprunter un modèle normatif (la communication critique) qui, comme nous l'avons vu, ne peut être compris que par la négation de l'inscription sociale, l'hypothèse de la table rase, de l'asocialité originaire. On nous propose ainsi une lecture limitant en quelque sorte les effets pervers d'un paradigme par l'introduction d'éléments opposés présents dans l'autre. Cette voie, lorsque pleinement reconnue, apparait d'ailleurs la plus pertinente à la lecture de la culture politique au travail actuellement dans les nouveaux mouvements sociaux. Nous nous interrogeons sur cette dimension en conclusion.

51. Voir C. Offe «New Social Movement...» (op. cit.) p. 856. Pour une analyse précédente où il ne percevait que des conflits systémiques dans le capitalisme avancé, voir C. Offe, "Political Authority and Class Structures - An Analysis of Late Capitalist Societies", International Journal of Sociology», Vol. 2, 1972-1973: 73-107. 


\section{Démocratie et communauté}

Un des héritages de la société industrielle, nous dit A. Mellucci ${ }^{52}$, est "l'image des mouvements sociaux aux caractères tragiques. (...) Au début des années quatre-vingt, poursuivra-til, presque rien semble avoir survécu de cette représentation épique». Son propre itinéraire de chercheur reprend, par ailleurs, le contour de ce cheminement. Préoccupé au départ de définir les conditions structurelles d'apparition des nouveaux mouvements sociaux, il tente plus récemment de comprendre comment des acteurs se regroupent dans un mouvement pouvant être représenté «comme une nébuleuse aux confins incertains et à la densité variable» ${ }^{33}$. Bref, un itinéraire comme plusieurs, qui parti à la recherche des nouvelles certitudes nées dans les sociétés du capitalisme avancé, abouti à la redécouverte du politique.

Voilà une piste d'interprétation possible de la matrice politique révélée par les nouveaux mouvements sociaux. J. Cohen remarque, par exemple, comment les acteurs, identifiés à la "nouvelle gauche» des années soixante, insistaient toujours pour inscrire leur action dans une vision «totalisante» et dans un projet "révolutionnaire» ${ }^{54}$. Aujourd'hui, souligne-t-elle, on est en présence d'un radicalisme auto-limitatif (self limiting radicalism). Une culture où les acteurs n'idéalisent plus le retour à une communauté indifférenciée et où ils luttent au nom de «l'autonomie», de la "pluralité» et de la «différence». Une matrice où ils n'inscrivent plus leurs revendications sur les normes et les valeurs à l'intérieur d'une perspective «fondamentaliste» (non négociable) mais, où ils acceptent d'inscrire leurs luttes dans le cadre formel de la démocratie et du marché. Un bilan relativement similaire donc à celui dressé, outre-atlantique,

\footnotetext{
52. A. Melucci, "The Symbolic Challenge..." (op. cit.), p. 809.

53. A. Melucci, "Mouvements sociaux, mouvements post-politiques» (op. cit.) p. 14.

54. J. Cohen, «Strategy or Identity:...» (op. cit.), p. 68.
} 
par L. Ferry et A. Renaut ${ }^{55}$ : après les illusions gauchistes ayant succédé immédiatement à Mai 68 , héritage tardif de la pensée historiciste révolutionnaire, des mobilisations fondées sur une reconnaissance de l'efficace du juridique, inscrites dans un horizon de possibles qui réanime la notion, longtemps considérée à gauche comme réactionnaire, d'une éthique de la responsabilité.

Pourtant, la dimension tragique des luttes sociales n'a pas disparu. C. Offe, nous l'avons vu, en dresse d'ailleurs une image qui fait ressortir une dimension beaucoup moins respectueuse des règles politiques formelles. Des acteurs qui refusent, la dichotomie classique droite/gauche, libéral/conservateur, les créneaux traditionnels de la représentation politique pour intervenir bruyamment, au nom de leur identité et autonomie, contre la centralisation du pouvoir. Des thèmes qui appellent peu à la nuance, parce que présentés sous l'égide de la survie de l'espèce humaine, de l'authenticité, de la position privilégiée parce que victime universelle. Des stratégies politiques qui font plutôt appels aux grands rassemblements, aux leaders médiatiques, aux gestes théâtraux et aux événements spectaculaires: très peu au processus électoral et à la délibération. Une matrice politique qui fera dire à Feller et Heller quẻ si la décennie des années soixante avait vu naître des mouvements pour la «liberté» ceux qui leur ont succédé sont des mouvements pour la «vie». Autrement dit, ils opposent au caractère «artificiel» de la modernité des considérations dites «naturelles» ou «organiques».

Campons bien l'antinomie ainsi présentée. D'une part, un paradigme qui perçoit dans l'abandon par les acteurs des grands projets totalisants, la confirmation des prémisses de la philosophie politique moderne: soit que le social est artefact, qu'il est le résultat de l'action politique. Une lecture qui par son relativisme

55. Voir les deux ouvrages précédemment cités, L. Ferry et $\mathrm{A}$. Renaut, La pensée $68, \ldots$, et $68-86$, Itinéraires... 
(anti-tragique), braque notre regard sur l'effervescence de nos sociétés, tout en nous laissant penaud devant l'indécision démocratique. Une grille qui éclaire un travail de discussion critique mené par des êtres humains se percevant comme individu, tout en ne pouvant que constater un mouvement, auquel il participe par ailleurs, d'atomisation et de développement de l'individualisme hédoniste. D'autre part, un paradigme qui voit dans l'éclatement des protestations actuelles une réaction diffuse d'identité contre l'instrumentalisation du monde. Inscrits au cœur des nouveaux rapports de pouvoir, ces mouvements sont des ripostes aux logiques technocratiques menées à partir d'une inscription singulière dans le social. Des acteurs alors doublement, dira-t-on, déterminés sociologiquement: par la structure d'ensemble de nos sociétés et par le lieu social particulier d'où l'on tire sa critique. "L'après socialisme» n'est plus indécis, il sera «anti-technocratique», comme le socialisme dans la société industrielle fut anti-capitalisme. Il puisera son contenu dans les situations objectives des communautés de base, des femmes, des jeunes, des minorités, des nécessités écologiques comme celles de la vie, etc. Une interprétation nous conduisant sur la route de la substance mais qui, en cherchant cette dernière dans les lois régissant la vie sociale, est conduite à nier le caractère subjectif (politique) de la construction de la cité. Une lecture du pluralisme actuel, à travers la revanche des inscriptions sociales, qui se réalise toutefois par un certain retour à la vision tragique de l'action politique. Autrement dit, des particularismes, se prétendant chacun fondé sur des positions "privilégiées», renouent avec un dogmatisme (communication iconographique) qui postule que la vérité doit s'imposer au delà de la démocratie (communication critique).

Quelle image en fait tirer de ce bilan paradigmatique des nouveaux mouvements sociaux? Ni l'une, ni l'autre des lectures ne nous a paru suffisante pour fonder à elle seule une matrice 
politique. D'autre part, comme elles reposent sur des postulats contradictoires, leur fusion s'avère impossible. Mais, peut-être plus important, ni l'un ni l'autre des paradigmes, n'annonce une brêche fondamentale dans la modernité politique. Le paradigme de l'individualisme démocratique, s'il reconnaît l'effervescence actuelle, en fait un simple moment de la révolution démocratique. Il nous invite à lire celle-ci en renouant avec les fondements modernes de la philosophie politique. Le paradigme de l'inscription sociale s'il inscrit la mouvance actuelle dans une rupture avec la société capitaliste/industrielle l'insère par contre dans une continuité théorique lorsqu'il s'agit d'analyser cette nouvelle société (inscription sociale des acteurs) et de préciser les fondements de ses exigences politiques (représentation des intérêts).

Pour le dire abruptement, chacune de ces lectures apparait déjà, depuis longtemps, constitutive de la modernité. Écoutons M. Gauchet nous en décrire le contour. «Le paradoxe constitutif de l'anthropologie des sociétés démocratiques, c'est que ces êtres pour la première fois reconnus en possession de droits originaires, inaliénables, antérieurs au fait collectif, pour la première fois ontologiquement autonomes, juridiquement maitres d'eux-mêmes, extérieurement indépendants pour la communauté où ils s'insèrent, sont aussi, et corrélativement, les premiers êtres à se découvrir asservis du dedans, intérieurement dépendants, et dépossédés d'euxmême par quelque chose ne venant de nulle part d'ailleurs que d'eux-mêmes " ${ }^{56}$. Autrement dit, l'idée démocratique d'apesanteur du social et l'idée sociologique d'une lourdeur de l'histoire, par notre inscription dans des communautés sont corrélatives de tout l'imaginaire moderne. Ce sont ces idées qui furent à la source des débats théoriques entre la raison pratique et la raison théorique, entre le constructivisme et l'historicisme, entre la liberté et le

56. M. Gauchet et G. Swann, Pratique de l'esprit humain, (op. cit.), p. 488. 
déterminisme. Cette même antinomie fut à l'œuvre aussi, dans les débats politiques opposant le libéralisme au conservatisme et au socialisme, comme ceux menés au nom des droits libertés ou des droits sociaux. Les nouveaux mouvements sociaux rééditent ainsi la brisure fondatrice sur laquelle s'est édifiée la modernité.

Que l'on ne puisse sortir aussi facilement de la modernité n'a rien de surprenant pour des sociétés qui, politiquement, ont refusé tout étalon extérieur donnant accès à un point hors-lemonde permettant de juger ce qui est rupture et ce qui ne l'est pas. Cela ne veut pas dire, pour autant, que l'émergence de nouveaux acteurs sociaux et les lectures qu'ils font naître sont sans signification en regard de la nouveauté politique. Les paradigmes dans lesquels ils s'insèrent, s'ils sont antinomiques, ne sont pas dans une relation de vrai ou de faux. Il nous semble, de toute évidence, qu'ils expriment plutôt deux sensibilités socio-politiques, difficilement réconciliables, mais constitutives des mêmes mouvements. Et c'est bien là, dans cette reformulation du rapport démocratie/communauté, que gît l'aspect novateur de cette matrice politique.

Expliquons nous. Historiquement, la tension entre les idées d'individualisme, de liberté, d'autonomie, de constructivisme contenues dans la notion de démocratie et, celles de déterminations historiques, d'aliénation et de solidarité réelle contenues dans la notion de communauté en est venue à recouper le projet politique libéral et le projet socialiste. Aujourd'hui, cette tension ne baliserait plus les frontières de deux courants politiques: les mouvements sociaux sont traversés par celle-ci. La gauche doit apprendre une nouvelle culture politique fondée sur les contradictions mêmes de la modernité: non uniquement sur l'un de ses pans. Accepter, par exemple, qu'aucune inscription sociale particulière ne nous donne la clef universelle de la lecture du social, tout en sachant que la position de sujet individuel démocratique est vide de 
contenu pratique. Apprendre difficilement donc à vivre entre le sens borné fourni par nos multiples asservissements sociaux et, l'indifférence universelle du citoyen.

Individu/société, privé/public, société-civile/État, éthique de la responsabilitéléthique de la conviction, droits de la personne/ droit sociaux, libéralisme/socialisme: c'est bien la tension révélée par cette série d'oppositions qui, aujourd'hui encore, est au travail dans la nouvelle culture politique révélée par les mouvements sociaux. Pas un travail qui demande une fusion des antinomies, plutôt une reformulation des frontières entre les éléments issus du paradigme de la démocratie et ceux issus du paradigme de la communauté. Un travail qui devrait nous conduire à reconnaître l'existence de principes opposés dans la réalité et nous apprendre à y faire face en créant l'espace où leur déploiement concurrentiel assure une mutuelle limitation. Un refus donc de saisir ces oppositions par la nécessité de l'élimination de l'un par l'autre, ou encore par un prétendu dépassement.

Pour ceux qu'une telle perspective chagrine, parce qu'elle n'offre pas l'horizon d'un monde radicalement autre, rappelons que c'est à l'intérieur de la reconnaissance de telles tensions que la modernité a fait voir son visage innovateur. Et, au contraire, lorsqu'on a cru posséder la clef assurant le monopole à l'un des éléments, ou celle assurant leur dépassement, on a brisé ces tensions créatrices... avec les catastrophes qu'on connaît. 